\title{
THE STRUCTURES OF ADJACENCY PAIRS OF FKIP UHN STUDENTS' IN ENGLISH CONVERSATION
}

\author{
Hilman Pardede ${ }^{1}$, Herman $^{2}$, Dumaris E. Silalahi ${ }^{3}$, Nguyen Van Thao ${ }^{*}$ \\ ${ }^{1,2,3}$ Faculty of Teacher Training and Education, English Education Department, Universitas HKBP Nommensen, \\ Indonesia \\ ${ }^{4}$ Faculty of Philology, Hanoi Pedagogical University 2, Vinh Phuc, Vietnam \\ hilman.pardede@uhn.ac.id ${ }^{1}$, herman@uhn.ac.id ${ }^{2}$, dumaris.silalahi@uhn.ac.id ${ }^{3}$,nguyenvanthao@hpu2.edu.vn ${ }^{4}$ \\ *Corresponding author: nguyenvanthao@hpu2.edu.vn
}

\section{ABSTRACT}

This study is aimed to investigate the structures of adjacency pairs in English conversation conducted by the students of faculty of teacher training and education (Fakultas Keguruan dan Ilmu Pendidikan/FKIP) at Universitas HKBP Nommensen (UHN). The subjects for conducting the SPP of Adjacency Pairs are ten, and for the FPP is one student. For turn-taking the subjects are fifteen students. The researchers apply a descriptive qualitative design in this study. The researchers observe what involved in the interaction, when, where, and how people interact based on Conversation Analysis (CA) approach. Because CA needs naturally occurring data, the researchers take the location of research outside classroom. The conversation outside classroom enacts an informal talk as what this research is about. The location outside classroom can be at the canteen, and benches around the campus. The results of the study found that there were ten structure of adjacency pair and turn-taking, they were: 1) The student's sequence of greeting-greeting is that the FPP is greeting and the FPP is greeting; 2) The construction of the students' APs in question-answer is : a) a question - answer, a question - a question; 3 ) The structure of compliment AP in student's conversation can be constructed as : a compliment - rejection, a compliment - a rejection in SPP (scaling down); 4) Offer-acceptance is composed: an offer of goods in FPP and an acceptance in SPP and an offer of service in FPP and an acceptance in SPP; 5) Invitation in student's conversation contains inserted sequence the acceptance response; 6) Current speaker selects next (CSSN) in student's conversation can be realized in two participants conversation like in all data in adjacency pair; 7) The CSSN allocation techniques is not always applicable in students' conversation; 8) Self-select (SS) in students' conversation is done as what is effective in English, but it is constrained by an overlapping talk; 9) Speaker continuation (SC) is shown by a long silence. The silence implies the development of topic or topic change. Last but not least, the researchers conclude that knowing the structure of adjacency pairs in conversation can help the speakers and listener to avoid and cope with all problems in speaking.

Keywords: Adjacency pairs, students' conversation, structures, conversation analysis, turn-taking

Article Received: 10 August 2020, Revised: 25 October 2020, Accepted: 18 November 2020

\section{INTRODUCTION}

Language is a purely human and non-instinctive method of communicating ideas, emotions, and desires by means of a system of voluntarily produced symbols [23]. From this definition, it is important to note that language may be seen as a form of communication unique to human beings. Sapir further points out that language does not exist apart from culture, that is, the socially inherited assemblance of practices and beliefs that determine the texture of our lives. From this condition it can be implied that speech activities, as the reflection of culture practised by a social group reveal the underlying attitudes in which the members of that social group are living.

When interaction occurs in a social context by using spoken language, the ones who are involved in the interaction need to be able to communicate verbally, in other words they have a verbal skill. Verbal skill is a linguistic skill, or spoken skill, which refers to the ability of speakers to use his language well. Every social group grants high status to members with good verbal skill. This verbal skill can been enacted in daily or mundane conversation of the members of society which constitute the basis for the further formal interaction. 
Furo presented four assumptions in analyzing action conducted during the conversation: 1) conversation is structurally organized, 2) conversation is jointly produced among participants, 3) conversation is contextual, and 4) conversation is locally managed [9]. These assumptions indicate that there are structures and process of turn-taking in conversation as well as units that build the turn-taking.

One of the approaches in analyzing conversation is conversation analysis (CA), which emerged in the pioneering researches of Sacks [13] into the structural organization of everyday language use, accounted by his friend Schegloff [12]. According to Schegloff's account, Sacks discovered some subtle ways in which callers to a suicide prevention center managed to avoid giving their names, as shown in the conversation below :
A : This is Mr. Smith, may I help you?
B : I can't hear you.
A : This is Mr. Smith.
B : Smith.

Sacks, as quoted by Hutchby and Woffit had observed that in the majority of cases if the person is taking the call within the organization started off by giving their name, then the suicidal person who was calling would be likely to give their name in reply [13]. But in one particular call, He noticed that the caller (B) as shown in the conversation above seemed to be having trouble with the name of the answerer. Then the agent who took the call found it difficult to get the caller's name. For him, the avoidance of giving one's name in the conversation by answering "I can't hear you" leads to the accomplishment of action or particular things given by an utterance. So, in this case the utterance is an action.

However, Sacks here emphasizes that "I can't hear you" is not always an expression representing the way one avoids giving his name. Rather he viewed the utterance as an action which is situated within specific context. He also observed that by the caller's "not hearing", he is able to set up a sequential trajectory in which the agent finds less opportunity to establish the caller's name without explicitly asking for it. Thereby the caller is able to begin the conversation by avoiding giving a name without actually refusing to do so.

Utterance as an action is also supported by Schegloff as he focused on action rather than a topic in talk-in interaction [24]. An utterance like "Would somebody like some more ice tea?" is better understood as "doing an offer" than as "about ice tea".

Conversation Analysis is derived from Ethnomethodology which is focused on the methods by which the group conducts conversation. Group here refers to society's members which are considered having intersubjectivity and common-sense knowledge realized in talk-in interaction in their daily life. Obviously, the member's knowledge meant by this method concerns with the member's knowledge of their ordinary affairs, knowledge that shows a sense of order in everyday conduct, and this is publicly displayed in activity which is going on.

Austin and Searle developed speech act theory from the basic insight that language is use, not just to describe the world, but it can perform an action [25]. The utterance "I promise to be there tomorrow" performs the act of promising, and the utterance "The grass is green" performs the act of asserting. An utterance can also perform more than one action as shown below.

Speaker : Can you pass the salt?

Hearer : ( pass the salt)

The first action is an act of questioning the ability of the hearer, and the second performs an act of requesting. This is what distinguishes utterance from sentence. In the case above there is only one sentence, that is, an interrogative sentence. But there are two utterances with two contexts. As Schiffrin put it, a sentence is neither a physical event nor a physical object, it is conceived of abstractly as a string of words put together by the grammatical rules of a 
language [25].

Of Sacks' observation on talk-in interaction, he really based his analysis on the naturally occurring data from which he did a turn by turn details of the conversation so that a robust analytical basis would be used to get a robust finding. What he observed then leads to the key insights which are treated on the methodological basis for conversation analysis. These key insights can be summarized below :

1. Talk - in interaction is systematically organized and deeply ordered.

2. The production of talk - in interaction is methodic.

3. The analysis of talk- in interaction should be based on naturally occurring data.

4. Analysis should not be constrained by prior theoretical assumption.

CA as a part of discourse occupies the functional domain of language by which in the former time this was not much in focus, especially in the context of classroom. Platt and Platt stated: "There has been a considerable shift in emphasis in linguistic research from phonology and morphology to syntax and semantics and from there on to an increased interest in the study of language in social context". There are two shifts of linguistic research in the quotation above, i.e., the one from phonology and morphology to syntax and semantics, and another one from syntax and semantics to study of language in social context. The study of language in social context or in a more specific term, a function - based study was less emphasized than the formal study of language [11].

The main basic units in CA are adjacency pairs and turn-taking. Adjacency pairs are sequences of two part actions, usually performed by different speakers, adjacently placed and differentiable into first pair-parts (FPP) and second pair-parts (FPP). The FPP is constrained by the SPP, e.g. greeting-greeting, question-answer, summon-answer, etc. Turn-taking is a process thorough which the party doing the talk of the moment is changed. Sacks et al. noted that in conversation not only does turn-taking occur, with one speaker tending to talk at a time, but turns are also taken with as little gap or overlap between them as possible [22].

From the one of the researcher's teaching experience, she found that there is a problematic issue of adjacency pairs in English conversation, that is, that the students did not respond the English compliment as what it should be, as seen in the following:

A : You are beautiful

B : No, I am not beautiful.

From the above conversation, A's compliment was rejected by B. It is not the case in English conversation, as compliment is not rejected, A's compliment is accepted in English conversation by an appreciation response such as, thank you. The second problem is that the turn-taking in English conversation done by the students do not always concord to the real rules of turn-taking in English conversation. For example, when the first rule of English turn-taking is: Current speaker selects next, the students' do not apply it in their conversation, as seen in the following.

John (to Henry) : What about our assignment?

Richard : It's difficult.

From the last two paragraphs it is found that there are some problematic issues that lead to the formulation of research problems of this study. One is that functional approach is a crucial point to see the structures of conversation of students, as conversation is structural. Structure here means conversation format based on CA theory. Then based on the structural approach in conversation, adjacency pairs are the basic units. The rejection of English compliment of students can be analyzed further in order to find the students' difficulties in English conversation as the mundane form rather than the formal one. Second, based on the local management of conversation it is necessary to analyze the turn-taking system of students' conversation. 


\section{LITERATURE REVIEW}

\subsection{Conversation}

The following parts talk about the characteristics of conversation as well as the assumption used conversation. Here the characteristic of conversation deal with the strength of mudane conversation used as data in conversation on Sack, Schegloff, and Jefferson study (in Furo, 2001). These parts also include adjacency pairs turn, and turn-taking.

\subsubsection{Characteristics Of Conversation}

As has been defined before that conversation is spontaneous talk in interaction among two or more participants in casual, informal settings of everyday life (Furo, 2001:25). This kind of conversation has also been touched by Goodwin and Heritage (1990) as ordinary and mundane conversation. Whereas listener freely alternate in speaking, and this occurs in formal setting. By those definitions we can direcly distinguish it from a talk that takes institutional setting as its context. For example, a conversation which occurs in a debate, seminar, adat ceremony, all of which the procedures: when, where, and how the participants' talk are not spontaneous.

In ordinary conversation or casual talk, there is no an arrangement who, where, and when to talk. All come spontaneously. Even though ordinary, and the general impression is that it is chaotic and disorderly, it is useful for conversation analysis which is based their work on ethnomethodological enquire, as expressed below:

Seeing the sense of ordinary activities means being able to see what people are doing and saying, and therefore one place in which one might begin to see how making sense is done in terms of the understanding of everyday talk (Sharrock and Anderson in Eggin and Slade, 1997:25).

When there was an invention of recording devices, and the willingness to study mundane conversation in depth, what people doing and saying in their everyday talk are actually highly organized and ordered.

According to Furo (2001) conversation treated as data in conversation analysis has three characteristics:

1. It reflected the communicative competence of the participant.

2. It is the most unmarked form of communication.

3. It reflected the interaction norms as well as the social system of the culture where it occurs.

This communicative competence as in 1) above, (Schiffrin in Furo, 2001) constitutes our tacit knowledge of the abstract rules of language, which is required both to produce sound/meaning correspondences between sounds, meaning forms in socially and culturally appropriate ways. Conversation requires participants knowledge of both linguistic and pragmatic ability, or show the way participant us language in interaction.

As the most unmarked form of communication, as in 2 ), conversation can be treated as the prototype of other forms of talk. Goodwin and Heritage (1990: 284) observed that ordinary conversation is the point of departure of more specialized communicative context. As it occurs in ubiquity, ordinary conversation has been familiar to the life of students in FKIP UHN. At this present time only talks in institutional setting, such as conversation in adat ceremony has been under investigation. Of its mundane nature, ordinary conversation may involve all people from all ranks, whereas institutional talks take limited participants, like only the married participants can participate in the conversation.

As conversation carried out in cultural and social context as in 3), the action done can reflect the identity of participants including interaction norms on social process in interpersonal relationships (Schiffirin, 1994). In this case, conversation can indicate the basic principles that govern the linguistic and non-linguistic behavior of the members of the society in which the principles are constituted.

\subsubsection{Assumptions In Conversation}

The four basic assumptions in conversation as discussed in chapter one (Furo, 2001: 24) are: 
1. Conversation is structurally organized.

2. Conversation is jointly produced among participants.

3. Conversation is contextual.

4. Conversation is locally managed.

Since conversation structurally organized and sequentially constrained (Goodwin and Heritage, 1990), there can be found structural approach, that is, adjacency pairs. This exemplifies structural organization as well as orderly sequence of interaction in conversation. Adjacency pairs give slot to the next position whether responded or not. When the first is not responded, the second would be noticeably absent, that leads to a repair actions.

As the joint production among participant, recipients show his or her intersubjective as the understanding and inferences of the speaker's utterance. Again, when recipients do not show his or her intersubjectivity, the speaker may reply with repair work in the next slot, which is called the third position repair (Schegloff in Furo, 2001).

Conversation is context dependent. This assumption means that conversation is shaped in context, the prior context (context-shaped) and the new context (context-renewing). In this way, context does not refer to social one such as participants' identities or situational settings but they are sequences of action and interpretation that emerge in the organization of conversation (Goodwin and Heritage, 1990)

The fourth assumption conversation is locally

\begin{tabular}{|l|l|l|}
\hline \multirow{2}{*}{1} & Taryn : How about some lunch ? & Invitation \\
\cline { 2 - 3 } & Jjay : Sound good. (stand up) & Acceptance \\
\hline
\end{tabular}

\begin{tabular}{|l|l|l|}
\hline 2 & $\begin{array}{l}\text { Taryn : How about some lunch ? } \\
\text { Jay : (pause) Uhh, better bot. } \\
\text { I've got to get this done by 2:00. } \\
\text { Thanks though. How's tomorrow? }\end{array}$ & $\begin{array}{l}\text { Invitation } \\
\text { refusal }\end{array}$ \\
\hline
\end{tabular}

There would be an expansion of adjacency pairs. This to ask the invited person if he has eaten yet. An is done by presequence. If a speaker wanted to invite someone for a dinner, it is reasonable for the speaker adjacency pairs usually a question-answer format come first, as in Example (3) below : managed, implies that turn-by-turn organization of conversation are analyzed. These occur in the turn-taking as presented by Sacks, Schegloff, and Jafferson (1974). They observed that turn exchanges are systematically realized with minimal gap and overlap because speakers take transition relevance place (TRPs).

\subsection{Adjacency Pairs}

We have noted above that structural view in interaction is related to adjacency pairs. That is a sequence of two utterances which are adjacent, produced by different speakers, ordered as a first pair part and second part, and typed, so that a first part requires a particular second part (Schegloff and Sacks in Schiffrin, 1994).

According to Tracy $(2002: 114)$ there are many kinds of adjacency pairs. Some pairs involve similar acts like greetings and goodbye, while others involve different acts, like invitations or offers followed by acceptances or refusals, and question followed by answer.

Below are two examples of common adjacency pairs in English taken from Tracy (2002:114). These adjacency pairs involve different acts. Example (1) accepts an invitation, and example (2) refuses an invitation. 


\begin{tabular}{|l|l|l|}
\hline \multirow{3}{*}{3} & Taryn : you eaten yet ? & Question \\
\cline { 2 - 3 } & Jay : No & Answer \\
\cline { 2 - 3 } & Taryn : How about some lunch ? & Invitation \\
\hline
\end{tabular}

Another expansion of adjecency pairs is done by determine if some condition applies that would make insertion sequences. Like presequences, insertion the conversationally preferred option possible. This is sequences involve an inserted adjecency pairs to presented in example (4) below :

\begin{tabular}{|l|l|l|}
\hline 4 & Taryn : How about some lunch ? & Invitation \\
\cline { 2 - 3 } & Jay : You got \$ 5 to lend me ? & Request \\
\cline { 2 - 3 } & Taryn : Yeah. & Grant \\
\cline { 2 - 3 } & Jay : Sounds good. & Acceptances \\
\hline
\end{tabular}

However, the notion of adjacency pair is not always the most usual sequence. It is possible for a question not to be answered by an answer, greeting by a greeting. When the answer is not forth coming it is noticeably absent (Schegloff in Tracy, 2002). In this case, it is possible for the speaker makes a repair. Schegloff (in Have, 1999) observed that from 500 instances of the telephone opening, one instance deviated from the common format summon-answer. As a matter of fact, this deviant is considered as noticeably absent. This can be seen in the following excerpt:

(police makes call)

Receiver is lifted, and there is one second pause

Police: Hello.

\section{Other : American Red-cross}

Police: Hello, this is police Headquartes, or Officer Stratton.

The common one is that a telephone ring is as a summons opens a conditional relevance for second part of a sequence, answer. If the answer is not forthcoming a summons can be reissued. In the above conversation, the police reissued a summons by saying, hello.

Another respect of adjacency pairs is preferences. Conversational preferences refer to structurally preferred second act for adjacency pairs that may take one of two forms (Tracy, 2002).

In offer, invitation or request, accepts are conversationally preferred to refusals. So, acceptance is a preferred action, and refusal in a dispreferred action. In English, conversationally dis-preferred act is always longer, more conversationally marked and elaborated. In example (2) before, the refusal of Jay as dispreferred action was not immediately given, but there was a pause before elaborating it.

It seem that conversational preference varies from culture to culture as what different languages tell us about the different concept of space in a certain culture (Duranti, 1997).

\section{METHODS}

The researchers apply a descriptive qualitative design in this study. A qualitative research refers to the meanings, concept, definitions, characteristics, metaphors, symbol, and description of things [2]. The word quality refers to the what, how, when, and where, and where of a thing-its essence and ambience. Concerning with the research being done, some of those concepts are applied as what to be observed are people interaction in conversation. The researchers observe what involved in the interaction, when, where, and how people interact based on CA approach.

Qualitative research has 3 characteristics [3]. It has natural setting, 2) The qualitative researches tend to analyze the data inductively, and 3) Meanings are of essential concerns in qualitative research. Natural setting in this study refers to spontaneous conversation conducted by students in campus, outside classroom. Inductive analysis is done in this research, and meanings in terms of action are applicable in CA approach. 
Conversation Analysis (CA) proposed by Sacks, Schegloff, and Jafferson (1974) enables us to analyze the recurring patterns in conversation and to find the ordeliness as well as the turn-by-turn of interaction in the students' conversation.

\subsection{Subject and Location of Research}

The subjects of research are the English students of FKIP Nommensen. The subjects for conducting the SPP of Adjacency Pairs are ten, and for the FPP is one student. For turn-taking the subjects are fifteen students.

The location of research is at the campus of FKIP Nommensen Pematangsiantar. Because CA needs naturally occurring data, the researchers take the location of research outside classroom. The conversation outside classroom enacts an informal talk as what this research is about. The location outside classroom can be at the canteen, and benches around the campus. The data are collected from these two places. The library is not taken as the location as it is considered as formal setting and not natural.

\subsection{Technique of Collecting Data}

Mundane or ordinary conversation is the data which is used in this research. Ordinary conversation is one that occurs in coffee counter, family houses, markets, and places that enacts such conversation. Institutional talk is not used in this research as it is not classified as natural-occuring data. There will be twenty-three data to be analysed. Six-teen data deals with adjacency pairs and seven data with turn-taking.

The data of research are in the form of conversation recorded in audio or tape-recording. The recordings were done in August 2019. Hutchby and Woofitt state that data in CA are the recorded interaction and transcribed one [13]. The transcription is seen as the representation of data while the tape is viewed as a reproduction. The transcription used in conjunction with the tape during analysis. In this case repeated listening to the original recording is central to the $\mathrm{CA}$ technique. The first step in doing transcription is to understand the transcription convention. The system developed by Sacks, Schegloff and Jefferson is in general used by conversation analyst [13]. Transcription procedures then are designed to make more and more accurate of naturally occuring talk.

As the data are naturally-occuring ones there are some difficulties found when the researcher collected them, especially the data for analysis of turn-taking in the location of research. The difficulties are:

1. To get the real natural data

2. To transcribe the data

3. To find the negative cases

\subsection{Data Analysis}

As has been said that there are four basic assumptions in CA: 1) conversation is structurally organized, 2) conversation is jointly produced among participants, 3 ) conversation is contextual, 4) conversation is locally managed. Two assumptions: 1) and 4), constitute a construct of the analysis in which assumption 1) deals with the structure of Adjacency Pairs, assumption 4) deals with turn by turn organization which conforms with the structure of turn taking.

The following are the construct of analysis [13]

1. Check the episode carefully in terms of turn-taking: the construction of turns, silence overlaps, etc ; make notes of any remarkable phenomena, especially on any disturbances in the fluent working of the turn-taking system.

2. Then look for sequences in the episode under review, especially adjacency pairs and their sequals.

\section{The data analysis procedures are summarized in the chart below.}




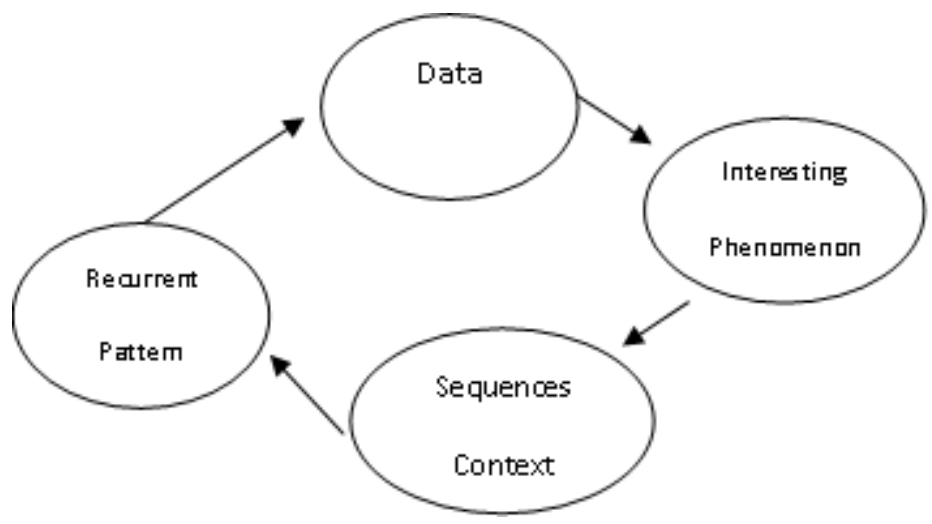

The figure reflects four basic procedures of analysing data. First the process of collecting data from which the second process derives, identifying interesting phenomenon. When for example, question-answer is an interesting phenomenon, the researcher has to find the context of sequences where it occurs. This phenomenon should emerge uniquely that makes it as new categorization as new finding. This is the third process. The fourth is that to find out the recurrent patterns to make the finding reliable.

\subsection{Validity}

In making the data valid, an accurate transcription is made by repeatedly listening and editing the recording. Repeated listening to the recorded data much influences the data categorization especially in turn-taking. When there is a doubt about the data, it would be verified to the speakers. So for the validity of data, the researcher applies the three steps: repeated-listening, transcribing, and verifying the data.

For the validity of findings, the researchers analyzed negative cases or the deviation with comparison to other opinions. Interviews are done with experts structurally and unstructurally by using notes.

For the ethical purpose, the informant consents is sought from the participants. In case the data are not yet sufficient, elicitation technique was done in which the researcher was involved.

\section{RESULTS}

After analyzing the data the researcher can construct some findings about the structure of APs and turn-taking as follows:
1. The student's sequence of greeting-greeting is that the FPP is greeting and the FPP is greeting. The most common of the sequence is that the FPP is in formal greeting,and the SPP is in formal too. There is a sequence of greeting in which the FPP contain formal greeting but the SPP is informal. This informal greeting results from a repair realized in informal of the silence sequence (data 2). It is also found that a respond to greeting can be in question (data 3 ).

2. The construction of the students' APs in question-answer is: a) a question in FPP and an answer in SPP (data 4), b) a question in FPP and a question in SPP in which the question as a response is considered greeting, as the question itself is a quasi-question (data 5), and c) a question in FPP and an answer in SPP which is preceded by inserted sequence (data 6).

3. The APs of complaint-rejection are composed of: a) a complaint from complainer in FPP and a rejection or dispreferred response or recomplaining in SPP (data 7), b) a complaint of the hearer's behaviour in FPP and a rejection in SPP (data 8), c) a complaint to the third party in FPP and an affiliation in SPP (data 9).

4. The structure of compliment AP in student's conversation can be constructed as : a) a compliment in FPP and rejection in SPP (data 10), b) a compliment is FPP and a rejection in SPP (scaling down) data (11), and c) a compliment in FPP and rejection inSPP (scaling down) preceded by inserted sequence (data 12). 
5. Offer-acceptance is composed: a) an offer of goods in FPP and an acceptance in SPP (data 13), and b) an offer of service in FPP and an acceptance in SPP (data 14).

6. Invitation in student's conversation contains inserted sequence the acceptance response. So the construction is : a) a compliment in FPP and an acceptance in SPP (acceptance preceded by inserted sequence) (data 15), and b) a compliment in FPP and an acceptance preceded by a rejection in the same sequence in SPP (data 16).

7. Current speaker selects next (CSSN) in student's conversation can be realized in two participants conversation like in all data in adjacency pair.

8. The CSSN allocation techniques is not always applicable in students' conversation; the speaker other than one who is selected may take the turn, and this always done by overlapping talk such as, terminal overlapping, continuers, and collaborative talk (data 17, 18, 19 successively)

9. Self-select (SS) in students' conversation is done as what is effective in Eglish, but it is constrained by an overlapping talk.

10. Speaker continuation (SC) is shown by a long silence. The silence implies the development of topic or topic change.

The findings above can be reconstructed in the following diagram

\section{GREETING-GREETING}

1.

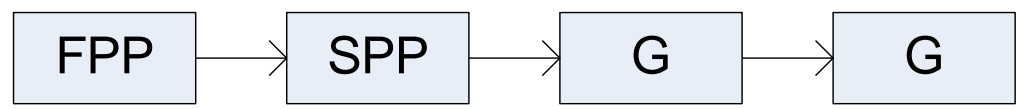

2.
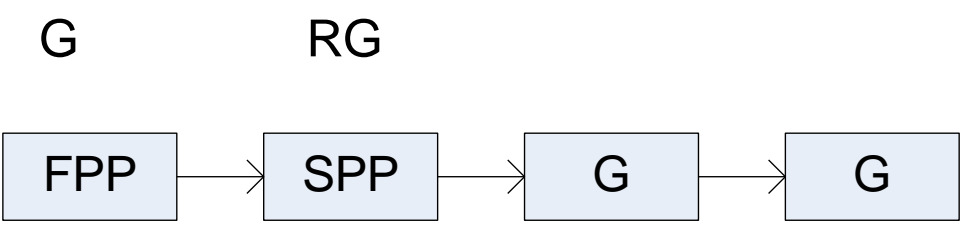

3.

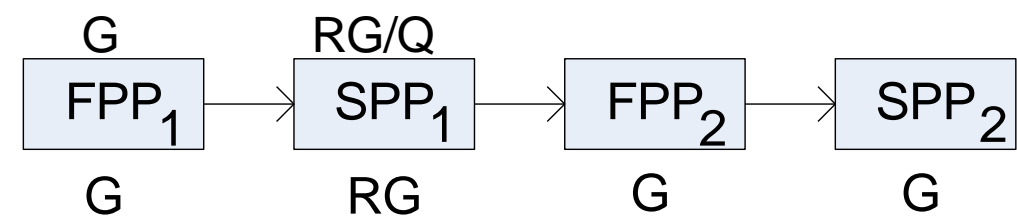

\section{QUESTION-ANSWER}

1.

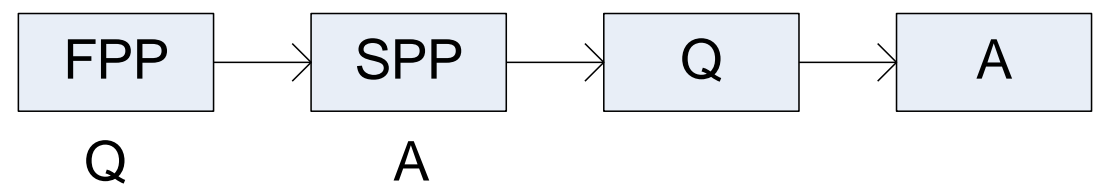


2.

3.
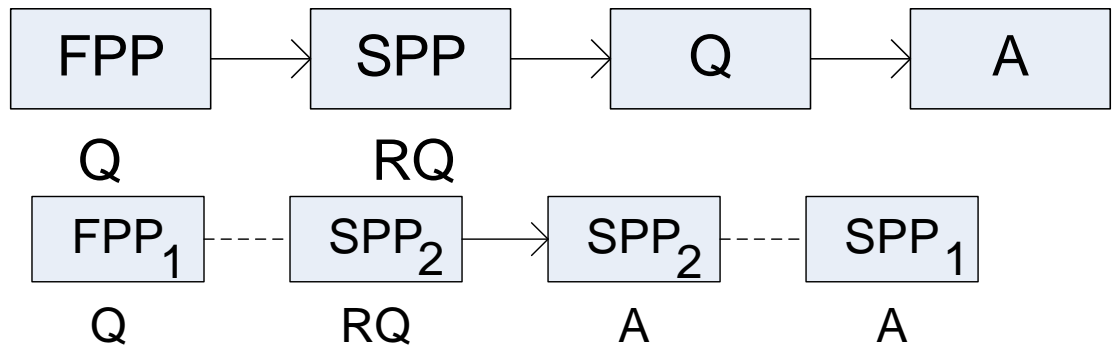

COMPLAINT- REJECTION

1.

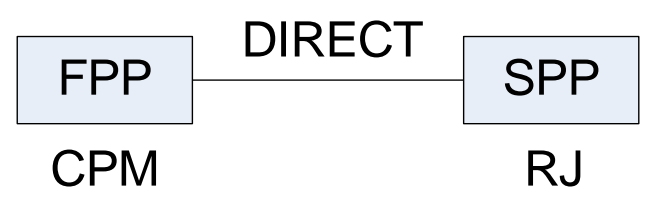

2.

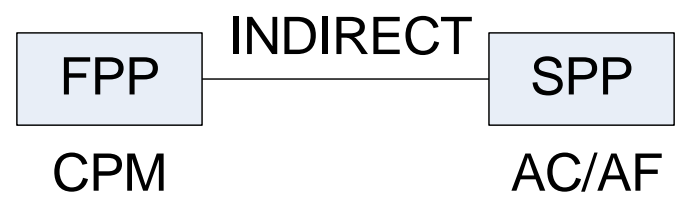

COMPLIMENT ACCEPTANCE

1.

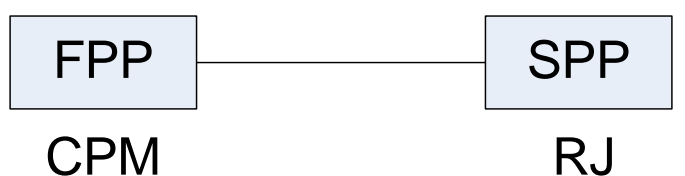

2.

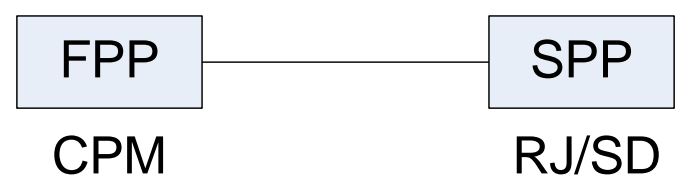

3.

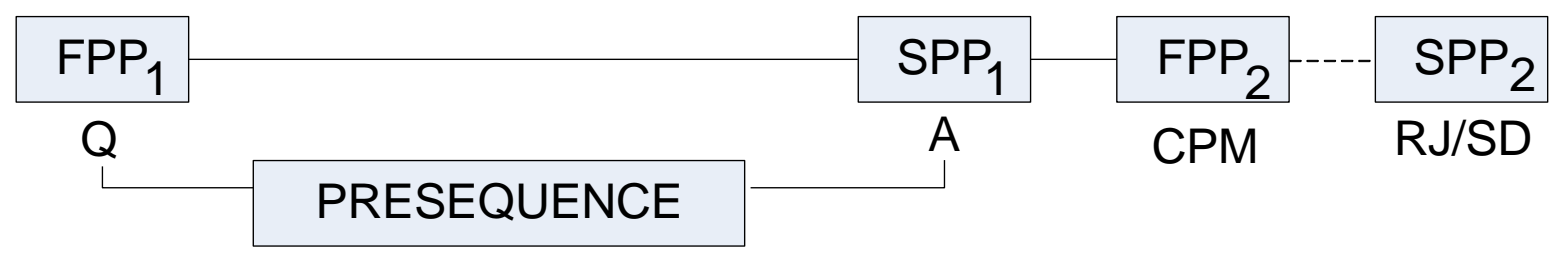


OFFER-ACCEPTANCE

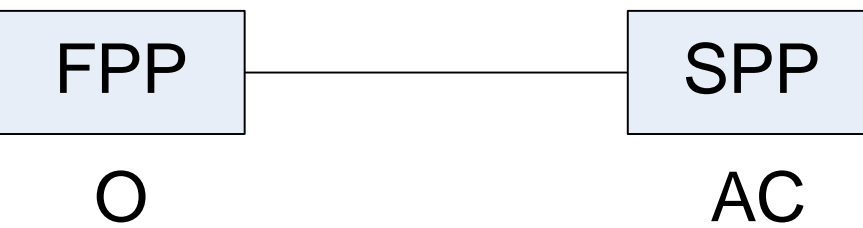

INVITATION-ACCEPTANCE

1.

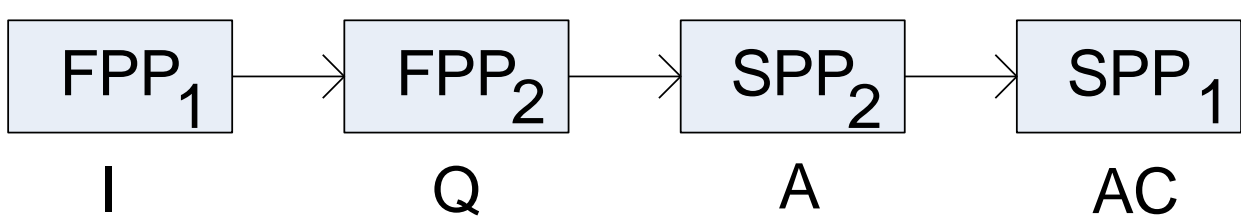

2.

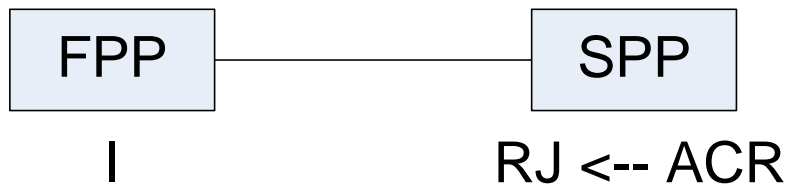

\section{STUDENTS' TURN TAKING}

1.

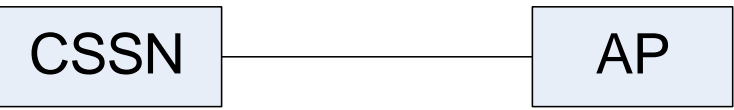

2.

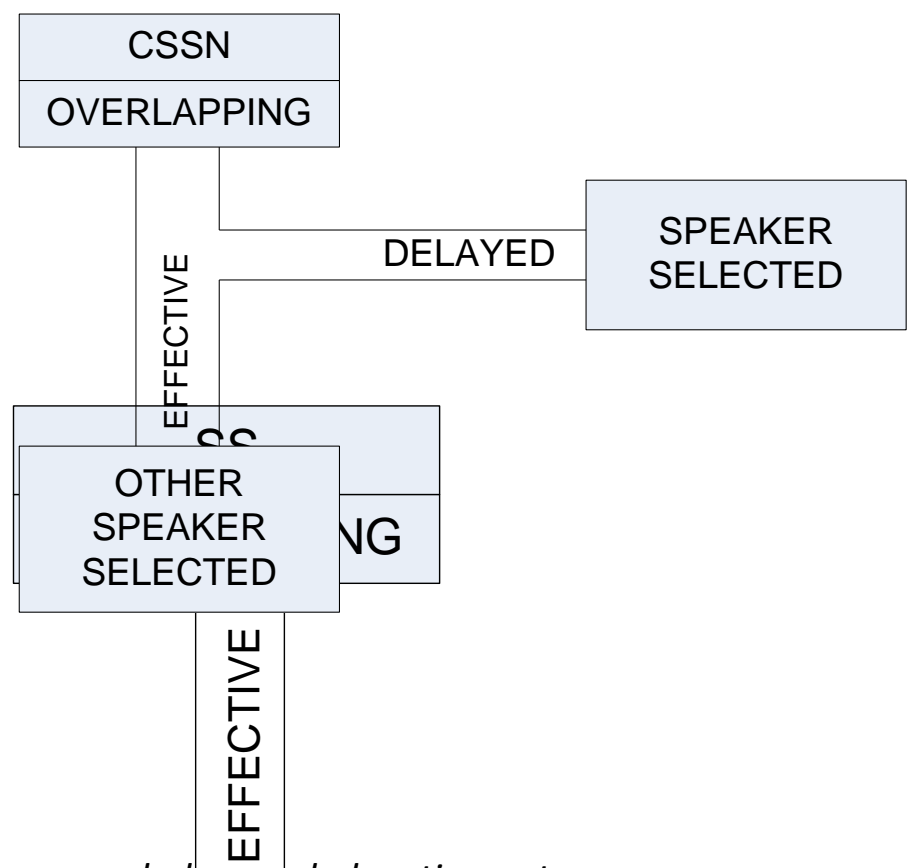

www.psycholoavandeducation.net

FIRST

STARTER 
4.

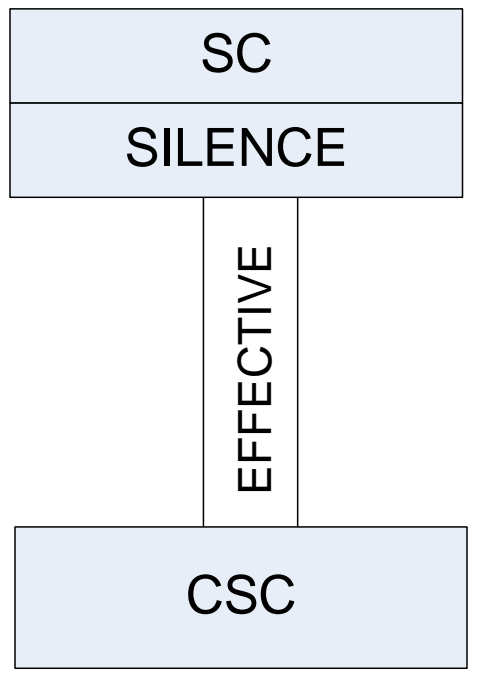

\section{DISCUSSION}

From the analysis and finding, greeting-greeting sequence is commonly practised in formal one. This is uncommon in English, as Schegloff and Sacks [33] have presented the structures of English greeting-greeting such as, hello-hi, hello-did you just get home?, good morning-hello. Whereas in Meitei language noted informal marker of greeting in conversation such as, nodding and smiling [1]. The response to greeting in question is also a unique phenomenon, as it is not commonly found in English.

For the question-answer sequence, the one which is considered out of its common form by which a question is responded by question, always occur in the students' conversation. In English when a question is responded question, this will be immediately answered.

The negative case in the student's AP of complaint-rejection is that it is not practised as what is done in English. In English the response to complaint is both rejection and affiliation. As what happens in this students' sequence, this will lead to a limited communicative competence of the students for a daily conversation.

The different response of the students' to compliment with that in English makes it problem for them when they get involved in interaction. They will always respond to compliment in rejection. They will never give a grant to ohers' compliment as what happens in their regional language.

Offer, on the other hand, is responded in acceptance. Whereas in English there are two responses, they are acceptance and rejection. What is offered in the students' conversation is both goods and service. For the students to have a normative conversation as what is practised in English they should adopt the English AP system.

The same as offer, invitation is responded by the students in acceptance, and it is preceded by an inserted AP. In English too there is such a sequence. The difference is that there are two responses to invitation in English, they are acceptance and rejection. The rejection is always elaborated. So this system also should be adopted by students in practising their daily conversation.

Both offer and invitation in the students' conversation have responses in acceptance in the SPP.

CSSN is considered to have constrained in overlapping talk such terminal overlapping, continuers, and collaborative talk. The students' overlap in the conversation is considered interruption in terminal overlap as it is not supportive to the speaker. Since it is botherful, students should keep aside this kind of interruption. 
Self-select technique implies that speakers should compete to be the first starter. This rule can result in overlapping talk as has been discussed above. From this it is logical to say that getting a turn can be a serious problem. We can imagine how a participant in a conversation, during the conversation, he never get even a turn because fails to compete with the others. In the data analysis all participants have at least a turn for each of them.

The students' conversation is also charecterized by a long silence when they practised speaker continuation (SC) technique. The silence never results in lapse until they finish the conversation. Actually the current speaker in SC gives an enough time for the other participants to take turn, but on the absence of turn the current speaker used the silence to extend or develop his turn. So the current speaker uses his intersubjectivity so that the conversation is accountable, in other words, as a daily conversation is about common sense, each speaker should have intersubjectivity so that the conversation goes smoothly.

\section{CONCLUSION}

Based on the previous analysis and discussions some conclusions can be drawn in the followings.

1. The students' conversation are structures in adjacency pairs such as: Greeting-Greeting, Question-Answer, Complaint-Rejection,

Compliment-Acceptance, Offer-Acceptance, Invitation-Acceptance.

2. The structures are operated in First Pair Part (FPP) and Second Pair Part (SPP). The additional structures are inserted sequence and presequence.

3. Greeting-Greeting is operated in three ways. First, greeting in FPP and response to greeting in SPP. Second, greeting in FPP and question as a response to greeting in SPP. Third, greeting in FPP1, silence as a response to greeting SPP1, greeting FPP2 and response to greeting in SPP2.

4. Question-Answer is operated in three ways. The first is question in FPP and an answer in SPP. The second is question in FPP and question as response to question in SPP. And the third is question in FPP1 question as response to question in FPP2, an answer in SPP2, and an answer as response to question in SPP1. FPP1 and SPP1 is the first AP, and FPP2 and SPP2 is the second AP.

5. There are two ways how Complaint-Rejection operated, they are: a) A complaint in FPP and a rejection in SPP. The AP is direct, b) A complaint in FPP and acceptance or affiliation in SPP. The $\mathrm{AP}$ is indirect.

6. Compliment-Acceptance is operated in three ways. First, a compliment in FPP and a rejection in SPP. Second, a compliment in FPP and rejection or scaling down in SPP. Third, A compliment in FPP2 and a rejection in SPP2 preceded by a presequence.

7. In Offer-Acceptance there is only one operation, offer in FPP and acceptance in SPP.

8. The Invitation-Acceptance is operated in two ways. First, invitation in FPP1, question in FPP2, an answer in SPP2 and an acceptance in SPP1. The second, invitation in FPP and an acceptance in SPP preceded by rejection in the same sequence.

9. There are some negative cases in AP of the students.

10. There are three allocation techniques of students' turn-taking, they are: a) CSSN, b) SS, and c) SC.

11. CSSN is not always applicable, and it is constrained by overlapping talk.

12. SS is a place to compete to get a turn.

13. SC is constrained by a long silence.

14. Silence in SC is a place to extend the current speaker turn.

15. There are some negative cases in students' turn-taking. 


\section{ACKNOWLEDGEMENTS}

This research is funded by Research and Community

Services Institutes (Lembaga Penelitian dan Pengabdian Masyarakat/LPPM) Universitas HKBP Nommensen, the Year 2020

\section{REFERENCES}

[1] Betholia, C. An analysis of Greeting and Leavetaking in Metei Speech. WWW. Ethnologue.com Manipur University, 2009

[2] Berg, Bruce L. Qualitative Research Methods. Boston : Allyn and Bacon, 1989

[3] Bogdan, Robert C. and Biklen, Sari Knopp. Qualitative Research for Education. Boston : Allign and Bacon Inc., 1982.

[4] Brown, Gillian and Jule, George. Discourse Analysis. Cambridge : Cambridge University Press. 1983

[5] Coulthard, Malcom. An Introduction to Discourse Analysis.London : Longman. 1977

[6] Cruz, Manuel Padilla. Pragmatics Applied to Language Teaching and Learning. Newcastle: Cambridge Scholars Publishing. 2009

[7] Duranti, Alessandro. Lingustic Anthropology. Cambridge : Cambridge University Press. 1997

[8] Eggins, Suzanne and Slade, Diana. Analyzing Casual Conversation. London : Cassel. 1997

[9] Ford, Cecilia E. and Sandra A. Thompson. Interactional units in conversation: syntactic, intonational, and pragmatic resources for the projection of turn completion. Interaction and grammar, ed. Elinor Ochs, Emanuel Schegloff, and Sandra A. Thompson, 134-184. Cambridge University Press. 1996

[10] Furo, Hiroko. Turn-Taking in English and Japanese. Routledge. 2001

[11] Goodwin, Charles. And Heritage, John. Conversation Analysis. Annual Reviews Inc. University of Australia. 1990.

[12] Halliday, M.A.K. An Introduction to Functional Grammar. London; Arnold. 1994

www.psychologyandeducation.net
[13] Have, Paul Ten. Doing Conversation Analysis. London: Sage Publication. 1999

[14] Herman, Murni, S. M., Sibarani, B. and Saragih, A. Structures of Representational Metafunctions of the "Cheng Beng" Ceremony in Pematangsiantar: A Multimodal Analysis. International Journal of Innovation, Creativity and Change., Volume 8, Issue 4, 2019. Retrieved from

https://www.ijicc.net/images/vol8iss4/8403_Her man_2019_E_R.pdf. 2019

[15] Hutchby, Ia and Woffitt, Roin. Conversation Analysis. Cambridge: Polity Press. 1999

[16] Hymes, Dell. Explorations in Ethnography of Speaking. In Bauman, R. and Sherzer, J. eds. 1974

[17] Juan Yu. Pedagogical Description of Compliment Response Exchanges in a British context for Chinese. The University of Eidenburgh. 2007

[18] Lerner, Gene, Conversation Analysis, Studies from the First Generation. Amsterdam: John Benjamins Publishing Company. 2004

[19] Markee, Numa. Conversation Analysis. London : Lawrence Erlbaum Associates Publishers. 2000

[20] Mathews, S.P.H. Oxford Concise Dictionary of Lingustic. Oxford: Oxford University Press. 1997

[21] McLaughin, Margaret L. Conversation How Talk Is Organized. California: Sage Publication. 1984

[22] Renkema, Jan. Discourse Studies. Amsterdam: John Benjamins Publishing Company. 1993

[23] Sacks, H. Schegloff, E. Jefferson, G. A Simplest Systematics For The Organization of TurnTaking For Conversation. Language. Vol 50. No 4. 1974

[24] Sapir, E. Language and Introduction To The Study of speech, New York: Harcourt, Brace 
and Company. 1921

[25] Schegloff, Emanuel. A. Sequences Organization in Interaction. Cambridge: Cambridge University Press. 2007

[26] Schiffrin, Deborah. Approaches to Discourse. Oxford : Blackwell. 1994

[27] Shotter, John. Conversation Realities. London : Sange Publication. 1993

[28] Tannen, Deborah. Conversation Style Analyzing Talk Among Friends. New Jersey : Albex Publishing Company. 1984

[29] Taylor, Talbot and Cameron, Deborah. Analysing Conversation Rules and Unit in the Structure of Talk. Oxford : Pengamon Press. 1987

[30] Tracy, Karen. Everyday Talk. New York : The
Guilford Press. 2002

[31] Traverso, Veronique. The Dilemmas of Third Party Complaint in Conversation Between Friends. Journalm of Pragmatics. 2872. 2008

[32] Van Dijk, Teun A. Discourse As Social Interaction. London : Sage Pulication. 1997

[33] Wood, Leuda. A. and Kroger, Ralf. O. Doing Discourse Analysis. California : Sage Publication. 2000

[34] William, Hosley. Membership Categorisation Analysis and Sequences and Meeting Talk. Cardiff University. 2001

[35] Zang Ping, On preferred and Dispreferred Second Turns in Interaction. Sino-US English Teaching Vol.4 No.12. 2007 